Volume 8, No.6, November - December 2019

International Journal of Advanced Trends in Computer Science and Engineering

Available Online at http://www.warse.org/IJATCSE/static/pdf/file/ijatcse35862019.pdf

https://doi.org/10.30534/ijatcse/2019/35862019

\title{
2-Fair Dominating Sets in Some Class of Graphs
}

\author{
Jayasree T G $^{1}$, Radha Rajamani Iyer ${ }^{2}$ \\ ${ }^{1}$ Adi Shankara Institute of Engineering and Technology, Kalady, Kerala, India. jayasreetg@gmail.com \\ ${ }^{2}$ Department of Mathematics, Amrita School of Engineering, Coimbatore, India.r_radhaiyer@cb.amrita.edu
}

\begin{abstract}
A set $D$ of vertices in a graph $G(V, E)$ is a dominating set of $\mathrm{G}$, if every vertex of $\mathrm{V}$ not in $\mathrm{D}$ is adjacent to at least one vertex in $D$. A dominating set $D$ of $G(V, E)$ is a $k$ - fair dominating set of $\mathrm{G}$, for $\mathrm{k} \geq 1$, if every vertex in $\mathrm{V}-\mathrm{D}$ is adjacent to exactly $\mathrm{k}$ vertices in $\mathrm{D}$. The $\mathrm{k}$ - fair domination number $\gamma_{\mathrm{kfd}}(\mathrm{D})$ of $\mathrm{G}$ is the minimum cardinality of a $\mathrm{k}$ - fair dominating set. In this article, we determine the k-fair domination number of some class of graphs for $\mathrm{k}=2$.
\end{abstract}

Key words: Fair Domination, Cartesian Product of Graphs, Square Graphs.

\section{INTRODUCTION}

Let $\mathrm{G}(\mathrm{V}, \mathrm{E})$ be a simple graph with vertex set $\mathrm{V}$ and edge set E. The order and size of $G$ are denoted by $n$ and $m$ respectively. For graph theoretic terminology we refer to Gary Chartrand and Ping Zhang [2] and [11, 12] Haynes et al. For any vertex $v \in V$, the open neighbourhood $\mathrm{N}(\mathrm{v})$ is the set $\{v \in V: u v \in E\}$, and the closed neighbourhood $\mathrm{N}[\mathrm{v}]$ is the set $N(v) \cup\{v\}$. For any $S \subseteq V, N(S)=\cup_{v \in S} N(v)$ and $N[S]=N(S) \cup S$.

A fair dominating set in graph $\mathrm{G}(\mathrm{V}, \mathrm{E})$ is a dominating set $\mathrm{D}$ such that all vertices not in $\mathrm{D}$ are dominated by same number of vertices from $\mathrm{D}$, that is, every two vertices not in $\mathrm{D}$ has same number of neighbours in $\mathrm{D}$. The fair domination number $\gamma_{\mathrm{fd}}(\mathrm{G})$ of $\mathrm{G}$ is the minimum cardinality of an fd-set.

A dominating set $D \subseteq V(G)$ is a 2 -fd set in $\mathrm{G}$, if for every two distinct vertices, $u, v \in(V-D),|N(u) \cap D|=|N(v) \cap D|=2$. That is, every two distict vertices not in $\mathrm{D}$ have exactly two neighbours from $\mathrm{D}$. The domination in graphs is one of the vital area in graph theory which has attracted many researchers because of its potentiality to solve and address many real life situations like in the communication, social network and in defense purpose to name a few. The following results are obvious.

Results: 1 . For complete graph $\mathrm{K}_{\mathrm{n}}, \gamma_{2 \mathrm{fd}}\left(\mathrm{K}_{\mathrm{n}}\right)=2$.

2. For complete bipartite graph $\mathrm{K}_{\mathrm{m}, \mathrm{n}}, \gamma_{2 \mathrm{fd}}\left(\mathrm{K}_{\mathrm{m}, \mathrm{n}}\right)=4$.

3. If $\mathrm{Sd}$ is a spider graph with $(2 n+1)$ vertices, then $\gamma_{2 \mathrm{fd}}\left(\mathrm{Sd}_{1,2 \mathrm{n}}\right)=\frac{\mathrm{n}}{2}+1$.
4. Let $P_{n}$, be a path of length $(n-1)$, then for $n \geq 3$, $\gamma_{2 \mathrm{fd}}\left(\mathrm{P}_{\mathrm{n}}\right)=\left\{\begin{array}{l}\left\lceil\frac{\mathrm{n}}{2}\right\rceil, \text { if } \mathrm{n} \text { is odd } \\ \frac{\mathrm{n}}{2}+1, \text { if } \mathrm{n} \text { is even }\end{array}\right.$.

5. Let $C_{n}$ be a cycle of length $n$, then $\gamma_{2 f d}\left(C_{n}\right)=$ $\left\lceil\frac{\mathrm{n}}{2}\right\rceil$, for $\mathrm{n} \geq 3$.

\section{SIERPINSKI TRIANGLE GRAPHS}

The Sierpinski triangle is a fractal named after Waclaw Sierpinski who described it in 1915. It is originally constructed as a mathematically generated pattern that can be reproduced at any magnification or reduction. An Algorithm for obtaining the Sierpinski triangle is given by Gabriele et.al in [1].

1. Start with an equilateral triangle with base parallel to the horizontal axis.

2. Shrink the triangle by $\frac{1}{2}$, make two copies, and position the three shrunk triangles so that each triangle touches each of the two other triangles at a corner.

3. Repeat step 2 with each of the smaller triangles.

As an initial step a triangle has to represented as a graph, three nodes connected by tree edges and this graph can be treated as generation zero of Sierpinski triangle graph. Next, on every edge of this triangle a node is placed and these new nodes are connected by edges. This forms a triangle consisting of four smaller triangles. The inner of the four triangles is considered 'dead' and no further "Sierpinski steps" will be performed here. The other three triangles are considered for further steps.

We have the following result.

2.1.Proposition: If $\mathrm{n}$ denotes the number of generations in Sierpinski triangle graph, then number of nodes is $\frac{3}{2} \times\left(1+3^{n}\right)$ and number of edges is $3^{\mathrm{n}+1}$.

2.2.Proposition: The domination number of Sierpinski graph is defined as $\gamma\left(S T^{n}\right)=\left\{\begin{array}{c}1, \text { for } n=0 \\ 2, \text { for } n=1 \\ 3^{n-1} \text { otherwise }\end{array}\right.$, where $n$ denotes the number of generations.

2.3.Theorem: If $\mathrm{n}$ denotes the number of generations in Sierpinski triangle graph then $\gamma_{2 f d}\left(S T^{n}\right)=\left\{\begin{array}{c}2, \text { for } n=0 \\ \frac{3}{2} \times\left[1+3^{n-1}\right] \text {, if } n \geq 1\end{array}\right.$. 


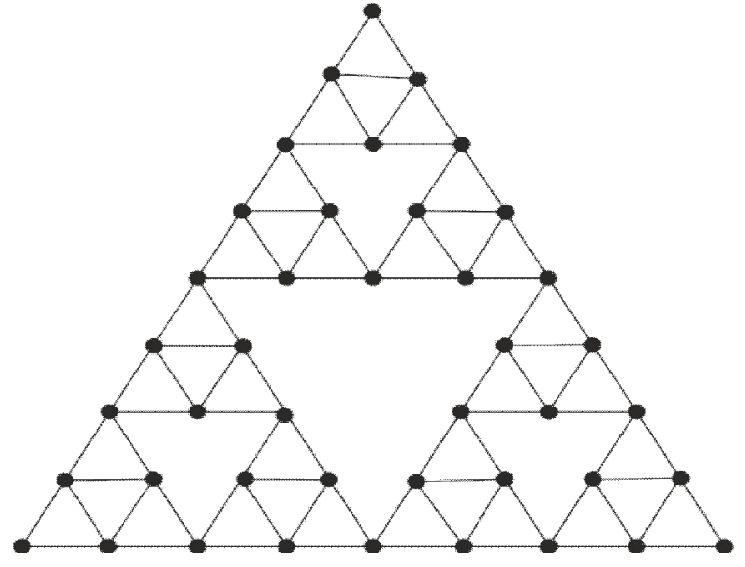

Figure 1: $S T^{n}$

Proof: The result can be proved by considering different generations of the graph. When $n=0$, the Sierpinski triangle is same as that of $C_{3}$. Hence $\gamma_{2 f d}\left(S T^{n}\right)=2$. The remaining can be proved using the method of mathematical induction.

Consider the case of $n=1$. This is Sierpinski triangle with 6 nodes and 9 edges. The graph can be dominated by 3 vertices as two fair domination. Hence the result is true for $n=1$. Let as assume that the theorem is true for $\mathrm{n}=\mathrm{m}$. That is $\gamma_{2 f d}\left(S T^{m}\right)=\frac{3}{2} \times\left[1+3^{m-1}\right]$.

Now we have to prove that the theorem is true for $n=m+1$. This case is the next generation of $n=m$. Here the number of nodes needed to dominate the entire the graph in two fair domination is

$$
\begin{gathered}
\gamma_{2 f d}\left(S T^{m+1}\right)=\left(\left(\frac{3}{2} \times\left[1+3^{m-1}\right]\right) \times 3\right)-3 \\
=\frac{1}{2}\left(9 \times\left[1+3^{n-1}\right]-6\right)=\frac{3}{2} \times\left(1+3^{m}\right) .
\end{gathered}
$$

The result is true for $n=m+1$. Hence by the method of mathematical induction, the theorem is true for all natural numbers. Therefore, the theorem is proved for all positive integers.

\section{TOTAL GRAPH OF $\mathrm{C}_{n}$}

Let $\mathrm{G}(\mathrm{V}, \mathrm{E})$ be a graph with vertex set $\mathrm{V}$ and edge set $\mathrm{E}$. The total graph of $\mathrm{G}$, denoted by $\mathrm{T}(\mathrm{G})$ is defined as follows. The vertex set of $\mathrm{T}(\mathrm{G})$ is $V(G) \cup E(G)$. Two vertices $\mathrm{u}, \mathrm{v}$ in $\mathrm{T}(\mathrm{G})$ are adjacent if any one of the following holds: (i) $\mathrm{u}, \mathrm{v}$ are in $\mathrm{V}(\mathrm{G})$ and $\mathrm{u}$ is adjacent to $\mathrm{v}$ in $\mathrm{G}$, (ii) $\mathrm{u}, \mathrm{v}$ are in $\mathrm{E}(\mathrm{G})$ and $\mathrm{u}$ is adjacent to $\mathrm{v}$ in $\mathrm{G}$, (iii) $\mathrm{u}$ is in $\mathrm{V}$ $(\mathrm{G})$, $v$ is in $E(G)$ and $u, v$ are incident in $G$.

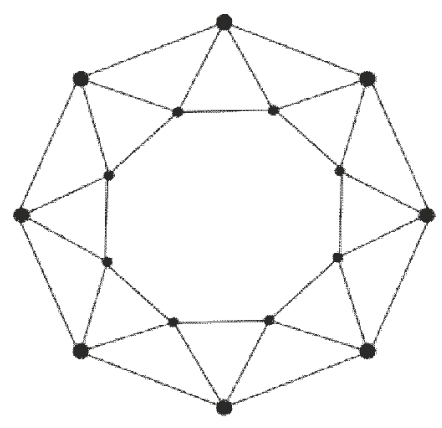

Figure 2: $\mathrm{T}\left(\mathrm{C}_{8}\right)$
3.1.Proposition: $\gamma\left(T\left[C_{n}\right]\right)=\left\lceil\frac{5 n}{3}\right\rceil$ for $n \geq 3$.

3.2.Theorem: For $n \geq 3, \gamma_{2 f d}\left(T\left[C_{n}\right]\right)=\left\lceil\frac{2 n}{3}\right\rceil$.

Proof: In $C_{n}$ every vertex dominates exactly three vertices. Hence in $T\left(C_{n}\right)$ every vertex dominates exactly 5 vertices because $T\left(C_{n}\right)$ is a 4 - regular graph. Let as consider the graph $T\left(C_{n}\right)$ as the union of two cycles $C_{n}$ and $C_{n}$ ' joined by edges. Take any vertex $v$ of $C_{n}$. Beginning with $\mathrm{v}$ proceed cyclically about these two cycles in some direction to cover all the $2 \mathrm{n}$ vertices. Let $\mathrm{D}$ be the set consisting of $\mathrm{v}$ and every vertices of both $C_{n}$ and $C_{n}$ ' such that they are dominated by exactly two vertices of $\mathrm{D}$. Now $\mathrm{D}$ become the two fair dominating set of the graph $\mathrm{T}(\mathrm{Cn})$ and $\gamma_{2 f d}\left(T\left[C_{n}\right]\right)=\left\lceil\frac{2 n}{3}\right\rceil$.

\section{SQUARE GRAPHS}

Let $\mathrm{G}(\mathrm{V}, \mathrm{E})$ be a simple graph with vertex set $\mathrm{V}$ and edge set $\mathrm{E}$. The $\mathrm{k}$-th power $\mathrm{G}^{\mathrm{k}}$ of the graph $\mathrm{G}$ is another graph that has the same set of vertices, but in which two vertices are adjacent when their distance in $\mathrm{G}$ is at most $\mathrm{k}$. The Square graph $\mathrm{G}^{2}$ of graph $\mathrm{G}$ is the graph that has the vertex set $\mathrm{V}$ in which two vertices are adjacent when their distance in $G$ is at most 2. Graph powers should be distinguished from the products of a graph with itself, which (unlike powers) generally have many more vertices than the original graph.

4.1.Proposition: For $n \geq 3, \gamma\left[\left(C_{n}\right)^{2}\right]=\left\lceil\frac{n}{5}\right\rceil$.

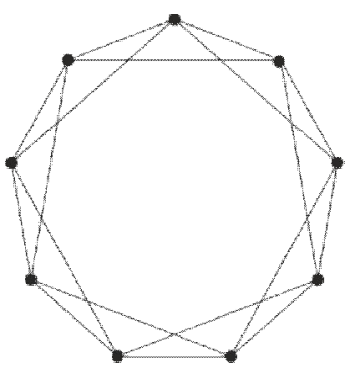

Figure 3: $\mathrm{C}_{9}{ }^{2}$

4.2.Lemma: $\gamma_{2 f d}\left[\left(C_{3}\right)^{2}\right]=\gamma_{2 f d}\left[C_{3}\right]=2$.

4.3.Theorem: $\gamma_{2 f d}\left[\left(C_{n}\right)^{2}\right]=\left\lceil\frac{n}{3}\right\rceil$ for $n \geq 3$.

Proof: Since $C_{n}$ is a two regular, $C_{n}{ }^{2}$ is a four regular graph. Hence every vertex of $\mathrm{C}_{n}{ }^{2}$ dominates exactly five vertices. Take a set $\mathrm{D}$ consisting of any vertex $\mathrm{v}$ of $\mathrm{C}_{\mathrm{n}}{ }^{2}$ and every third vertex of $\mathrm{C}_{\mathrm{n}}{ }^{2}$, starting with $\mathrm{v}$ and proceed cyclically in some direction. Then every vertex of the graph is dominated exactly twice by the vertices of $\mathrm{D}$. So D is a two fair dominating set of $\mathrm{C}_{\mathrm{n}}{ }^{2}$ and $\gamma_{2 f d}\left[\left(C_{n}\right)^{2}\right]=\left[\frac{n}{3}\right]$. Hence the proof.

\section{CARTESIAN PRODUCT OF GRAPHS}

The Cartesian product of two graphs $\mathrm{G}$ and $\mathrm{H}$, is a graph $G \square H$, [9] with vertex set $V(G \square H)=V(G) \times V(H)$ and edge set $E(G \cdot H)=\left\{\left(\left(x_{1}, y_{1}\right),\left(x_{2}, y_{2}\right)\right):\left(x_{1}, x_{2}\right) \in\right.$ $E(G)$ with $y_{1}=y_{2}$ or $\left(y_{1}, y_{2}\right) \in E(G)$ with $\left.x_{1}=x_{2}\right\}$. Hamed et.al.[3] have studied the perfect dominating sets in the Cartesian product of cycles of prime order. A necessary and sufficient condition for the existence of an efficient dominating set in the Cartesian product of two cycles has explained by T.Tamiz 
Chelvam et.al [10]. Here we try to find the general formula for Cartesian product of cycles of any order.

\subsection{Theorem: $\gamma_{2 \mathrm{fd}}\left(\mathrm{C}_{3} \square \mathrm{C}_{3}\right)=3$.}

Proof: The graph $\boldsymbol{C}_{\mathbf{3}} \square \boldsymbol{C}_{\mathbf{3}}$ has 9 vertices and 18 edges. Let as name the vertices as $\left\{v_{11}, v_{12}, v_{13}, v_{21}, v_{22}, v_{23}, v_{31}, v_{32}, v_{33}\right\}$. Then the vertices $\left\{v_{11}, v_{22}, v_{33}\right\}$ is enough to dominate the remaining six vertices as two fair domination as among these three vertices any two fairly dominates the remaining six. Hence $\gamma_{2 \mathrm{fd}}\left(\mathrm{C}_{3} \square \mathrm{C}_{3}\right)=3$.

5.2.Lemma: $\gamma_{2 f d}\left[C_{n}\right]=\left\lceil\frac{n}{2}\right\rceil$ for $n \geq 3$.

5.3. Theorem: $\gamma_{2 \mathrm{fd}}\left(\mathrm{C}_{\mathrm{n}} \bullet \mathrm{C}_{\mathrm{n}}\right)=\mathrm{n} \times\left\lceil\frac{\mathrm{n}}{2}\right\rceil$ for $n \geq 4$.

Proof: The graph $\mathrm{C}_{\mathrm{n}} \square \mathrm{C}_{\mathrm{n}}$ can be considered as n cycles arraged one over the other all are connected again by cycle $C_{n}$. But by the above lemma each $\mathrm{C}_{\mathrm{n}}$ can two fair dominated by $\left[\frac{\mathrm{n}}{2}\right]$ vertices. Hence we have $\gamma_{2 \mathrm{fd}}\left(\mathrm{C}_{\mathrm{n}} \square \mathrm{C}_{\mathrm{n}}\right)=\mathrm{n} \times \gamma_{2 f d}\left[C_{n}\right]=\mathrm{n} \times\left\lceil\frac{\mathrm{n}}{2}\right\rceil$ for $n \geq 4$.

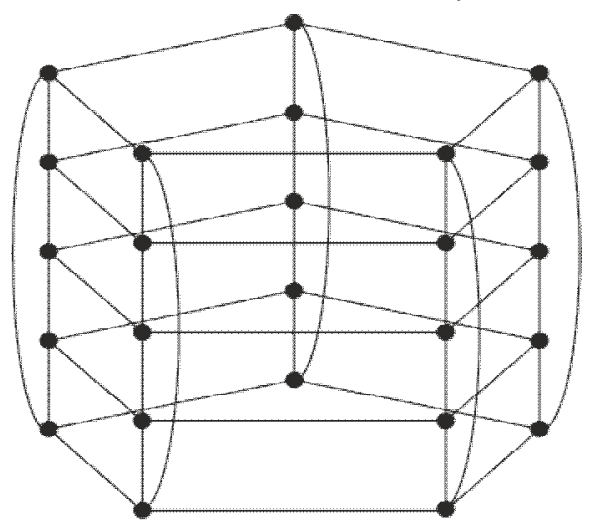

Figure 4: $C_{5} \square C_{5}$

5.4.Theorem: For any $<n$, and $m, n \geq 4$, $\gamma_{2 f d}\left(C_{m} \boxminus C_{n}\right)=\left\{\begin{array}{c}n \times\left[\frac{m}{2}\right], \text { if } m \text { is even and } n \text { is odd } \\ m \times\left[\frac{n}{2}\right], \text { otherwise }\end{array}\right.$.

Proof: For any $m<n$, the graph $\mathrm{C}_{\mathrm{m}} \square \mathrm{C}_{\mathrm{n}}$ can be treated as $\mathrm{n}$ cycles arranged one over the other which are connected again by $\mathrm{m}$ cycles. Since we are looking for the minimum number, from the above theorem and lemma the result follows.

5.5.Theorem: Let $G=H \circ K$, be the corona product of the graphs $\mathrm{H}$ and $\mathrm{K}$ obtained by taking one copy of $\mathrm{H}$, called the centre graph, $|\mathrm{V}(\mathrm{H})|$ copies of $\mathrm{K}$, called the outer graph, such that for each vertex $\mathrm{u}_{\mathrm{i}} \in \mathrm{V}(\mathrm{H})$, adjacent to every vertex of $\mathrm{i}^{\text {th }}$ copy of $\mathrm{K}$. Let $K \in \mathcal{K}$, where $\mathcal{K}$ consists of the set $\left\{\mathrm{P}_{3}, \mathrm{~K}_{\mathrm{n}}\right\}$, for $n \geq 2$. Then $\gamma_{2 \mathrm{fd}}(\mathrm{G})=$ $2 \times|\mathrm{V}(\mathrm{H})|$, for any graph $\mathrm{H}$.

Proof: Since the graph $\mathrm{G}$ is the corona product of the graph $\mathrm{H}$ and $\mathrm{K}$, where $K \in \mathcal{K}$, by the definition itself we can see that every vertex of $\mathrm{H}$ can be dominated by $\mathrm{K}$ as 2 fair domination. Hence the result follows.

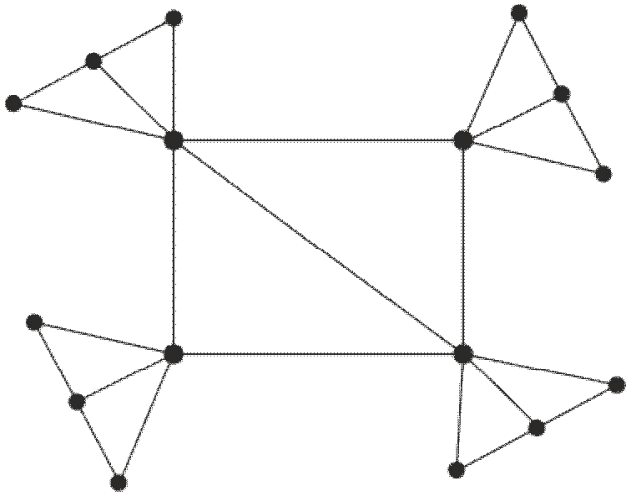

Figure 5: $K_{4}-\{e\} \circ P_{3}$

5.6. Theorem: Let $G=H \circ K$, be the corona product of the graphs $\mathrm{H}$ and $\mathrm{K}$. When $\mathrm{K}$ is the complete bipartite graph $\mathrm{K}_{\mathrm{m}, \mathrm{n}}$, for $m, n \geq 2$.. Then $\gamma_{2 \text { fd }}(\mathrm{G})=3 \times|\mathrm{V}(\mathrm{H})|$ for any graph $\mathrm{H}$.

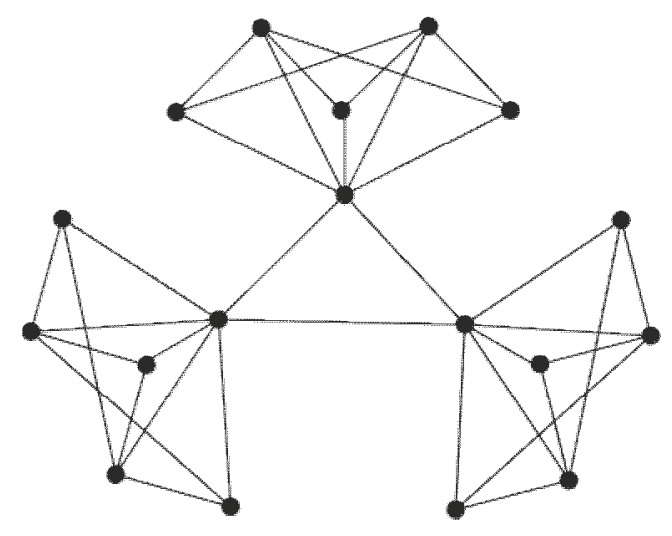

Figure 6: $C_{3} \circ K_{2,3}$

Proof: By the construction of complete bipartite graph two vertices can dominate the entire graph $\mathrm{K}$. Along with these two, the one vertex from the centre graph dominates one copy of the corona graph. There exists $|\mathrm{V}(\mathrm{H})|$ such copies. So the graph $\mathrm{G}$ requires $3 \times|\mathrm{V}(\mathrm{H})|$ vertices to dominate the entire graph by 2 - fair domination. Hence the result.

\section{CONCLUSION}

We have discussed generally in this work and in article [6] some particular cases of fair domination for certain class of graphs. Also we have defined the inverse cases of fair domination and discussed some cases in article [5].

\section{ACKNOWLEDGEMENT}

We have tried our best to achieve maximum quality for this work. Constant support from the members of editorial board and reviewers has made this possible. The comments and suggestions from their end have contributed for the improvement of this work. We express our sincere gratitude to them. 


\section{REFERENCES}

[1] Gabriele Taentzer, Enrico Biermann, et.al., 'Generation of Sierpinski Triangles: A Case Study for Graph Transformation Tools'.

[2] Gary Chartrand, Ping Zhang, 'Introduction to Graph Theory', Tata McGraw-Hill Edition (2006).

[3] Hamed Hatami, Pooya Hatami, 'Perfect dominating sets in the Cartesian products of prime cycles', The Electronic J.Comb., 2007, 14(1).

[4] Harshita Chaurasiya, Dr. Shivanath Ghosh, "Performace Evaluation of Energy-Efficient Cluster Based Algorithms in Wireless Sensor Networks", International Journal of Advanced Trends in Computer Science and Engineering, Vol.7, No.5, pp.77-81, 2018.

https://doi.org/10.30534/ijatcse/2018/03752018

[5] Jayasree T G, Radha R Iyer, 'New Parameters on Inverse Domination', International Journal of Mechanical Engineering and Technology, Vol.10, Issue.3, April 2019, pp. 1111- 1116.

[6] Jayasree T G, Radha Rajamani Iyer, '1-fd sets in some class of graphs', International Journal of Advanced Trends in Computer Science and Engineering, Vol. 8, No. 3, 2019, pp. 610 - 614.

https://doi.org/10.30534/ijatcse/2019/44832019

[7] M. M. Akbar Ali, S. Panauappan, 'Cycle multiplicity of total graph of Cn, Pn, and $K_{1}, \mathbf{n}$ ', International Journal of Engineering, Science and Technology, Vol.2, No.2, 2010, pp.54-58.

https://doi.org/10.4314/ijest.v2i2.59138

[8] P.Jeyanthi, G. Hemalatha, B. Davvaz, 'Total Domination Subdivision Number in Strong Product Graph', American Journal of Applied Mathematics and Statistics, 2014, Vol.2, No.4, 216-219.

https://doi.org/10.12691/ajams-2-4-7

[9] R. R. Iyer, A. Ganesan, 'The regular number of a graph', Journal of Discrete Mathematical Sciences and Cryptography, 2012. https://doi.org/10.1080/09720529.2012.10698371

[10] T. Tamizh Chelvam, Sivagnanam Mutharasu, 'Efficient domination in Cartesian Products of Cycles'. Journal of Advanced Research in Pure Mathematics, Online ISSN 1943-2380, Vol.3, Issue. 3, 2011, pp. 42-49.

https://doi.org/10.5373/jarpm.594.100910

[11] T. W. Haynes, S. T. Hedetniemi, P. J. Slater 'Domination in Graphs: Advanced Topics'. Marcel Dekker, New York (2000).

[12] T. W. Haynes, S. T. Hedetniemi, P. J. Slater 'Fundamentals of Domination in Graphs'. Marcel Dekker, New York (2000). 